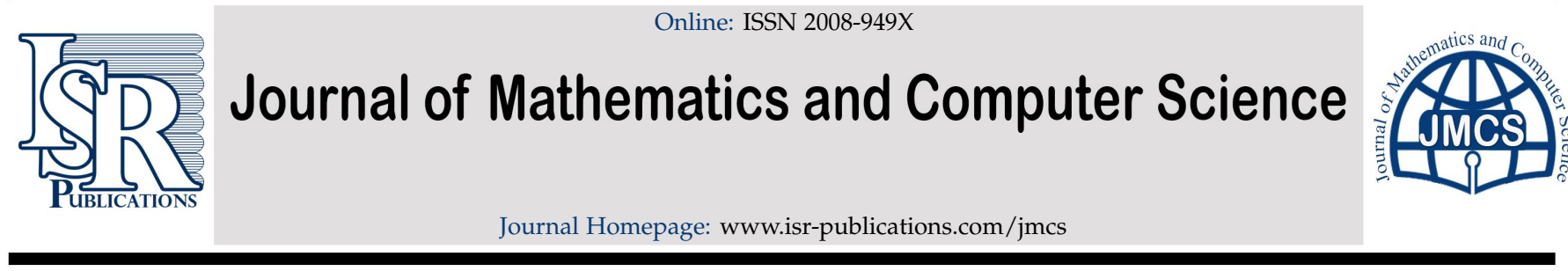

\title{
Determinants, inverses, norms, and spreads of skew circu- lant matrices involving the product of Fibonacci and Lucas numbers
}

\author{
Yunlan Wei ${ }^{\mathrm{a}, \mathrm{b}}$, Yanpeng Zheng ${ }^{\mathrm{c}, *}$, Zhaolin Jiang ${ }^{\mathrm{a}, *}$, Sugoog Shon ${ }^{\mathrm{b}}$ \\ a School of Mathematics and Statistics, Linyi University, Linyi 276000, China. \\ ${ }^{b}$ College of Information Technology, The University of Suwon, Hwaseong-si, 445-743, Korea. \\ ${ }^{c}$ School of Automation and Electrical Engineering, Linyi University, Linyi 276000, China.
}

\begin{abstract}
In this paper, we investigate the invertibility of $n \times n$ skew circulant matrix involving the product of Fibonacci and Lucas numbers, whose determinant and inverse can be expressed by the $(n-1)^{\text {th }}, n^{\text {th }},(n+1)^{\text {th }},(n+2)^{\text {th }}$ product of Fibonacci and Lucas numbers. Some norms and bounds for the spread of these matrices are given, respectively. In addition, we generalize these results to skew left circulant matrix involving the product of Fibonacci and Lucas numbers. Finally, several numerical examples are illustrated to show the effectiveness of our theoretical results.
\end{abstract}

Keywords: Determinant, inverse, norm, spread, Fibonacci number, skew circulant matrix.

2010 MSC: 15A09, 15A15, 15B05, 11B39, 65F40.

(C)2020 All rights reserved.

\section{Introduction}

Circulant matrices play a vital role in nonlinear control systems, preconditioners, signal processing, image processing, Toeplitz matrix problems and various differential equations [3, 7, 8, 19, 23, 26-28]. Jin et al. [14] proposed the GMRES (Generalized Minimal Residual) method with the Strang-type blockcirculant preconditioner for solving singular perturbation delay differential equations. Next, Pelletier and Cooperstock [22] put forward preconditioners based on circulant operators for speeding up the solution of edge-preserving image super resolution problems. Besides, circulant matrices have an important application in the optimization design of digital filters. Narasimha [21] decomposed linear convolution into a sum of skew circulant convolutions, which perform filtering procedure in approximately half of computational cost for real signals. Shortly afterwords, based on circulant, Toeplitz and shift circulant matrices, Daher et al. [1] developed a fast algorithm for optimal design of block digital filters, which reduced the computational complexity of the design process as well as decreased the memory requirements. Better

\footnotetext{
*Corresponding author

Email addresses: zhengyanpeng0702@sina.com (Yanpeng Zheng), jzh1208@sina.com (Zhaolin Jiang)
}

doi: $10.22436 /$ jmcs.020.01.08

Received: 2019-07-30 Revised: 2019-08-06

Accepted: 2019-08-27 
yet, Fu et al. [4] proposed a skew circulant filter, which achieves the above advantages of [1] and even greater efficiency with only approximately half of the computational cost for the real signals.

On the other hand, Fibonacci sequences are shining stars in the vast array of integer sequences and Fibonacci numbers appear in many unexpected places, such as electrical networks, nature (earth, optics and botany), even music and so on. For centuries, they amaze engineers with their charm and applications such as electrical engineering, geometry, graph theory, physics, physiology, psychology, neurophysiology, biology, chemistry, stock market trading, water treatment, snow plowing, and trigonometry $[6,16,17]$.

Recently, some authors have done some research on circulant matrices involving some famous numbers about their determinants, inverses, some norms, and spreads [2, 5, 10, 11, 13, 18, 20, 24, 25]. Recently, Zheng and Shon [30] studied the exact determinants and inverses of generalized Lucas skew circulant type matrices, while Jiang and Hong [12] gave that of Tribonacci r-circulant type matrices. What's more, AhmetÍpek [9] investigated an improved estimation for spectral norms of circulant matrices with classical Fibonacci and Lucas number entries.

We present, therefore, the results on the combination of skew circulant matrix, skew left circulant matrix, Fibonacci numbers and Lucas numbers. More specifically, we study the invertibility, determinant, multiple norms, lower and upper bounds for the spread of these matrices, which are going to have potential to be useful for realistic application.

The main aims we study in the paper are as follows. Fibonacci numbers $F_{n}$ and Lucas numbers $L_{n}$ are often defined recursively [16] as

$$
F_{n}=F_{n-1}+F_{n-2} \text {, where } F_{1}=1, F_{2}=1 \text { and } L_{n}=L_{n-1}+L_{n-2} \text {, where } L_{1}=1, L_{2}=3 \text {. }
$$

The product $\mathfrak{F}_{n}$ of Fibonacci and Lucas numbers satisfies the following recurrence relations [20]:

$$
\mathfrak{F}_{\mathfrak{n}+2}=3 \mathfrak{F}_{\mathfrak{n}+1}-\mathfrak{F}_{\mathfrak{n}}, \text { where } \mathfrak{F}_{1}=1, \mathfrak{F}_{2}=3 .
$$

The sequence $\left\{\mathfrak{F}_{n}\right\}$ is also given by the formula

$$
\mathfrak{F}_{n}=\frac{\alpha^{n}-\beta^{n}}{\alpha-\beta}
$$

where $\alpha$ and $\beta$ are the roots of the characteristic equation $x^{2}-3 x+1=0$. The first few values of the sequence are given in the following table:

\begin{tabular}{c|ccccccccc}
$\mathrm{n}$ & 1 & 2 & 3 & 4 & 5 & 6 & 7 & 8 & $\cdots$ \\
\hline $\mathfrak{F}_{n}$ & 1 & 3 & 8 & 21 & 55 & 144 & 377 & 987 & $\cdots$
\end{tabular}

The product $\mathfrak{F}_{n}$ of Fibonacci and Lucas numbers recurrence relations play a vital role in the following paper.

Consider a skew circulant matrix involving the product of Fibonacci and Lucas numbers

$$
\operatorname{SCirc}\left(\mathfrak{F}_{1}, \mathfrak{F}_{2}, \ldots, \mathfrak{F}_{n}\right)=\left(\begin{array}{cccc}
\mathfrak{F}_{1} & \mathfrak{F}_{2} & \ldots & \mathfrak{F}_{\mathrm{n}} \\
-\mathfrak{F}_{\mathrm{n}} & \ddots & \ddots & \vdots \\
\vdots & \ddots & \ddots & \mathfrak{F}_{2} \\
-\mathfrak{F}_{2} & \ldots & -\mathfrak{F}_{\mathrm{n}} & \mathfrak{F}_{1}
\end{array}\right)_{\mathrm{n} \times \mathrm{n}}
$$

Besides, define a skew left circulant matrix involving the product of Fibonacci and Lucas numbers

$$
\operatorname{SLCirc}\left(\mathfrak{F}_{1}, \mathfrak{F}_{2}, \ldots, \mathfrak{F}_{\mathfrak{n}}\right)=\left(\begin{array}{cccc}
\mathfrak{F}_{1} & \ldots & \mathfrak{F}_{\mathfrak{n}-1} & \mathfrak{F}_{\mathfrak{n}} \\
\vdots & . \cdot & . \cdot & -\mathfrak{F}_{1} \\
\mathfrak{F}_{\mathfrak{n}-1} & . \cdot & . \cdot & \vdots \\
\mathfrak{F}_{\mathfrak{n}} & -\mathfrak{F}_{1} & \ldots & -\mathfrak{F}_{\mathfrak{n}-1}
\end{array}\right)_{\mathfrak{n} \times \mathfrak{n}}
$$




\section{Preliminaries}

In this section, we list some lemmas that play a vital role in the proofs of main theorems.

Lemma 2.1. Let $\left\{\mathfrak{F}_{n}\right\}$ be the product of Fibonacci and Lucas numbers. Then

$$
\begin{aligned}
& \text { (i) } \sum_{i=1}^{n} \mathfrak{F}_{i}=2 \mathfrak{F}_{n}-\mathfrak{F}_{n-1}-1, \\
& \text { (ii) } \sum_{i=1}^{n} \mathfrak{i} \mathfrak{F}_{i}=(n+2) \mathfrak{F}_{n+1}-(n+3) \mathfrak{F}_{n}-2, \\
& \text { (iii) } \sum_{i=1}^{n} \mathfrak{F}_{i}^{2}=\frac{\mathfrak{F}_{n+1}^{2}-\mathfrak{F}_{n}^{2}-8 n-7}{5}, \\
& \text { (iv) } \sum_{i=1}^{n} \mathfrak{F}_{i} a^{i}=\frac{\mathfrak{F}_{n} a^{n+2}-\mathfrak{F}_{n+1} a^{n+1}+a}{a^{2}-3 a+1}, \quad\left(a \neq \frac{3 \pm \sqrt{5}}{2}\right) .
\end{aligned}
$$

Proof.

(i) According to (1.1)

$$
\sum_{i=1}^{n} \mathfrak{F}_{\mathfrak{i}}=\mathfrak{F}_{1}+\mathfrak{F}_{2}+\cdots+\mathfrak{F}_{\mathfrak{n}}=\mathfrak{F}_{1}+\left(3 \mathfrak{F}_{1}-\mathfrak{F}_{0}\right)+\cdots+\left(3 \mathfrak{F}_{n-1}-\mathfrak{F}_{n-2}\right)=2 \sum_{i=1}^{n} \mathfrak{F}_{\mathfrak{i}}-2 \mathfrak{F}_{\mathfrak{n}}+\mathfrak{F}_{\mathfrak{n}-1}+\mathfrak{F}_{1}-\mathfrak{F}_{0} .
$$

It is easy to check that

$$
\sum_{i=1}^{n} \mathfrak{F}_{\mathfrak{i}}=2 \mathfrak{F}_{n}-\mathfrak{F}_{n-1}-1
$$

(ii) From (1.1), we also get that

$$
\begin{aligned}
\sum_{i=1}^{n} i \mathfrak{F}_{i}=\mathfrak{F}_{1}+2 \mathfrak{F}_{2}+\cdots+n \mathfrak{F}_{n} & =\mathfrak{F}_{1}+2\left(3 \mathfrak{F}_{1}-\mathfrak{F}_{0}\right)+\cdots+n\left(3 \mathfrak{F}_{n-1}-\mathfrak{F}_{n-2}\right) \\
& =2 \sum_{i=1}^{n} i \mathfrak{F}_{i}+\sum_{i=1}^{n} \mathfrak{F}_{i}-(2 n+1) \mathfrak{F}_{n}+(n+1) \mathfrak{F}_{n-1}+\mathfrak{F}_{1}-2 \mathfrak{F}_{0}
\end{aligned}
$$

By (2.5) and (2.6), we have

$$
\sum_{i=1}^{n} \mathfrak{i} \mathfrak{F}_{i}=(n+2) \mathfrak{F}_{n+1}-(n+3) \mathfrak{F}_{n}-2
$$

(iii) Let $X_{i}=\left(\begin{array}{cc}\mathfrak{F}_{\mathfrak{i}-1} & \mathfrak{F}_{i} \\ \mathfrak{F}_{i} & \mathfrak{F}_{\mathfrak{i}+1}\end{array}\right)$. By Lemma 1 in [29], for $i \geqslant 1$, we get

$$
\left|X_{i}\right|=\mathfrak{F}_{i-1} \mathfrak{F}_{i+1}-\mathfrak{F}_{i}^{2}=-1 .
$$

For $\left\{\mathfrak{F}_{n}\right\}$, the following equation is satisfied,

$$
\mathfrak{F}_{i}=\frac{\mathfrak{F}_{i+1}+\mathfrak{F}_{i-1}}{3}
$$

Therefore, using (2.7), we obtain that

$$
\sum_{i=1}^{n} \mathfrak{F}_{i}^{2}=\sum_{i=1}^{n}\left(\frac{\mathfrak{F}_{i+1}+\mathfrak{F}_{i-1}}{3}\right)^{2}=\frac{\sum_{i=1}^{n} \mathfrak{F}_{i+1}^{2}+\sum_{i=1}^{n} \mathfrak{F}_{i-1}^{2}+2 \sum_{i=1}^{n} \mathfrak{F}_{i+1} \mathfrak{F}_{i-1}}{9}
$$




$$
\begin{aligned}
& =\frac{\sum_{\mathfrak{i}=1}^{n} \mathfrak{F}_{\mathfrak{i}+1}^{2}+\sum_{\mathfrak{i}=1}^{n} \mathfrak{F}_{\mathfrak{i}-1}^{2}+2 \sum_{\mathfrak{i}=1}^{n} \mathfrak{F}_{\mathfrak{i}}^{2}-2 \mathfrak{n}}{9} \\
& =\frac{4 \sum_{i=1}^{n} \mathfrak{F}_{i}^{2}+\mathfrak{F}_{\mathfrak{n}+1}^{2}-\mathfrak{F}_{\mathfrak{n}}^{2}-\mathfrak{F}_{1}^{2}+\mathfrak{F}_{0}^{2}-2 \mathfrak{n}}{9} .
\end{aligned}
$$

Hence

$$
\sum_{i=1}^{n} \mathfrak{F}_{i}^{2}=\frac{\mathfrak{F}_{\mathfrak{n}+1}^{2}-\mathfrak{F}_{\mathfrak{n}}^{2}-8 \mathrm{n}-7}{5}
$$

(iv) Let

$$
S_{n}=\sum_{i=1}^{n} \mathfrak{F}_{i} a^{i}=\mathfrak{F}_{1} a+\mathfrak{F}_{2} a^{2}+\cdots+\mathfrak{F}_{n} a^{n}
$$

From (1.1) and (2.8), we get

$$
\left(a^{2}-3 a+1\right) S_{n}=\mathfrak{F}_{n} a^{n+2}-\mathfrak{F}_{n+1} a^{n+1}+a .
$$

Thus

$$
S_{n}=\frac{\mathfrak{F}_{n} a^{n+2}-\mathfrak{F}_{n+1} a^{n+1}+a}{a^{2}-3 a+1}, \quad\left(a \neq \frac{3 \pm \sqrt{5}}{2}\right) .
$$

Lemma 2.2. Let the form of the matrix $\mathcal{H}=\left[h_{i j}\right]_{i, j=1}^{n-2}$ be as follows:

$$
h_{i j}= \begin{cases}1+\mathfrak{F}_{n+1}, & i=j, \\ -\mathfrak{F}_{n}, & i=j+1 \\ 0, & \text { otherwise. }\end{cases}
$$

Then $\mathcal{H}^{-1}=\left[h_{i, j}^{\prime}\right]_{i, j=1}^{n-2}$ is given by

$$
h_{i j}^{\prime}= \begin{cases}\frac{\mathfrak{F}_{n}^{i-j}}{\left(1+\mathfrak{F}_{n+1}\right)^{i-j+1}}, & i \geqslant j \\ 0, & i<j .\end{cases}
$$

Proof. Let $e_{i j}=\sum_{k=1}^{n-2} h_{i k} h_{k j}^{\prime}$. For $i<j, e_{i j}=0$. For $i=j, e_{i i}=h_{i i} h_{i i}^{\prime}=\left(1+\mathfrak{F}_{n+1}\right) \cdot \frac{1}{1+\mathfrak{F}_{\mathfrak{n}+1}}=1$. For $i \geqslant j+1$, we obtain

$$
e_{i j}=\sum_{k=1}^{n-2} h_{i k} h_{k j}^{\prime}=h_{i, i-1} h_{i-1, j}^{\prime}+h_{i i} h_{i j}^{\prime}=\frac{-\mathfrak{F}_{n} \cdot \mathfrak{F}_{n}^{i-j-1}}{\left(1+\mathfrak{F}_{n+1}\right)^{i-j}}+\frac{\left(1+\mathfrak{F}_{n+1}\right) \cdot \mathfrak{F}_{n}^{i-j}}{\left(1+\mathfrak{F}_{n+1}\right)^{i-j+1}}=0 .
$$

We get $\mathcal{H H}^{-1}=I_{n-2}$, where $I_{n-2}$ is $(n-2) \times(n-2)$ identity matrix. Similarly, we can verify $\mathcal{H}^{-1} \mathcal{H}=$ $I_{n-2}$. Thus, the proof is completed.

Lemma 2.3 ([5]). Let the orthogonal skew left circulant matrix $\Delta=\operatorname{SLCirc}(1,0,0, \ldots, 0)$. Then

$$
\operatorname{SCirc}\left(a_{1}, a_{2}, \ldots, a_{n}\right)=\Delta \operatorname{SLCirc}\left(a_{1}, a_{2}, \ldots, a_{n}\right) .
$$

Lemma 2.4 ([15]). Let $A=\operatorname{SLCirc}\left(a_{1}, a_{2}, \ldots, a_{n}\right)$ be a skew left circulant matrix and $n$ be odd. Then

$$
\lambda_{j}= \pm\left|\sum_{k=1}^{n} a_{k} \omega^{\left(j-\frac{1}{2}\right)(k-1)}\right|, \quad\left(j=1,2, \cdots, \frac{n-1}{2}\right), \quad \lambda_{\frac{n+1}{2}}=\sum_{k=1}^{n}\left|a_{k}(-1)^{k-1}\right|,
$$

where $\omega=\cos \frac{2 \pi}{n}+i \sin \frac{2 \pi}{n}=e^{\frac{2 \pi i}{n}}$ and $\lambda_{j}\left(j=1,2, \cdots, \frac{n-1}{2}, \frac{n+1}{2}\right)$ are the eigenvalues of $A$. 
3. Determinant, inverse, norms, and spread of skew circulant matrix involving the product of Fibonacci and Lucas numbers

In this section, let $A_{n}=\operatorname{SCirc}\left(\mathfrak{F}_{1}, \ldots, \mathfrak{F}_{n}\right)$ be an $n \times n$ skew circulant matrix. We do some work on the determinant and inverse of $A_{n}$. Meanwhile, we also investigate the properties of skew circulant matrices involving the product of Fibonacci and Lucas numbers, such as their norms, lower and upper bounds for their spread.

\subsection{Determinant and inverse of skew circulant matrix involving the product of Fibonacci and Lucas numbers}

In this subsection, we first obtain an expression for the determinant of $A_{n}$ in the following Theorem 3.1. Afterwards, we prove that $A_{n}$ is invertible for every positive integer $n$ in Theorem 3.2, and then we compute the inverse of $A_{n}$ in Theorem 3.3.

Theorem 3.1. Let $A_{n}=\operatorname{SCirc}\left(\mathfrak{F}_{1}, \ldots, \mathfrak{F}_{n}\right)$ be a matrix as in (1.3) for a positive integer $n$. Then

$$
\operatorname{det} A_{n}=\frac{\mathfrak{F}_{n}^{n}+\left(1+\mathfrak{F}_{n+1}\right)^{n}}{1-\mathfrak{F}_{n-1}+\mathfrak{F}_{n+1}}
$$

where $\mathfrak{F}_{\mathrm{n}}$ is the $\mathrm{n}^{\text {th }}$ product of Fibonacci and Lucas numbers.

Proof. Clearly, $\operatorname{det} A_{1}=1, \operatorname{det} A_{2}=10$, and $\operatorname{det} A_{3}=558$ meet (3.1). Under the circumstances $(n>3)$, construct two matrices

$$
\Sigma=\left(\begin{array}{ccccccc}
1 & 0 & \ldots & \ldots & \ldots & \ldots & 0 \\
3 & \vdots & & & & . \cdot & 1 \\
-1 & \vdots & & & . \cdot & . \cdot & -3 \\
0 & \vdots & & . \cdot & . \cdot & . \cdot & 1 \\
\vdots & \vdots & . \cdot & . \cdot & . \cdot & . \cdot & 0 \\
\vdots & 0 & . \cdot & . \cdot & . \cdot & . \cdot & \vdots \\
0 & 1 & -3 & 1 & 0 & \ldots & 0
\end{array}\right)_{n \times n}
$$

and

$$
\Omega_{1}=\left(\begin{array}{cccccc}
1 & 0 & 0 & \ldots & \ldots & 0 \\
0 & \left(\frac{\mathfrak{F}_{\mathfrak{n}}}{1+\mathfrak{F}_{\mathfrak{n}+1}}\right)^{n-2} & \vdots & & . \cdot & 1 \\
\vdots & \vdots & \vdots & . \cdot & . \cdot & 0 \\
\vdots & \left(\frac{\mathfrak{F}_{n}}{1+\mathfrak{F}_{\mathfrak{n}+1}}\right)^{2} & 0 & . \cdot & . \cdot & \vdots \\
\vdots & \frac{\mathfrak{F}_{\mathfrak{n}}}{1+\mathfrak{F}_{\mathfrak{n}+1}} & 1 & . \cdot & & \vdots \\
0 & 1 & 0 & \ldots & \ldots & 0
\end{array}\right)_{\mathfrak{n} \times \mathfrak{n}}
$$

Multiplying $A$ by $\Omega_{1}$ and $\Sigma$ from right and left, respectively,

$$
\Sigma A_{n} \Omega_{1}=\left(\begin{array}{ccccccc}
\mathfrak{F}_{1} & l_{n}^{\prime} & \mathfrak{F}_{n-1} & \mathfrak{F}_{n-2} & \cdots & \cdots & \mathfrak{F}_{2} \\
0 & l_{n} & \mathfrak{F}_{n-2} & \mathfrak{F}_{n-3} & \cdots & \cdots & \mathfrak{F}_{1} \\
\vdots & 0 & \mathfrak{F}_{1}+\mathfrak{F}_{n+1} & 0 & \cdots & \cdots & 0 \\
\vdots & \vdots & -\mathfrak{F}_{n} & \ddots & \ddots & & \vdots \\
\vdots & \vdots & 0 & \ddots & \ddots & \ddots & \vdots \\
\vdots & \vdots & \vdots & \ddots & \ddots & \ddots & 0 \\
0 & 0 & 0 & \cdots & 0 & -\mathfrak{F}_{n} & \mathfrak{F}_{1}+\mathfrak{F}_{n+1}
\end{array}\right)_{n \times n}
$$


where

$$
l_{n}^{\prime}=\sum_{k=1}^{n-1} \mathfrak{F}_{k+1}\left(\frac{\mathfrak{F}_{n}}{1+\mathfrak{F}_{n+1}}\right)^{n-1-k}, \quad l_{n}=1+3 \mathfrak{F}_{n}+\sum_{k=1}^{n-2} \mathfrak{F}_{k}\left(\frac{\mathfrak{F}_{n}}{1+\mathfrak{F}_{n+1}}\right)^{n-1-k}
$$

We further simplify the above results using (2.4) as follows:

$$
\begin{aligned}
l_{n}^{\prime} & =\frac{-1+\mathfrak{F}_{n-1}+\mathfrak{F}_{n}\left(1+\mathfrak{F}_{n+1}\right)^{n-2}}{\left(1-\mathfrak{F}_{n-1}+\mathfrak{F}_{n+1}\right)\left(1+\mathfrak{F}_{n+1}\right)^{n-2}}, \\
l_{n} & =\frac{\mathfrak{F}_{n}^{n}+\left(1+\mathfrak{F}_{n+1}\right)^{n}}{\left(1-\mathfrak{F}_{n-1}+\mathfrak{F}_{n+1}\right)\left(1+\mathfrak{F}_{n+1}\right)^{n-2}} .
\end{aligned}
$$

So it holds that

$$
\operatorname{det}\left(\Sigma A_{n} \Omega_{1}\right)=l_{n}\left(1+\mathfrak{F}_{n+1}\right)^{n-2}=\frac{\mathfrak{F}_{n}^{n}+\left(1+\mathfrak{F}_{n+1}\right)^{n}}{1-\mathfrak{F}_{n-1}+\mathfrak{F}_{n+1}}
$$

Clearly, det $\Sigma=\operatorname{det} \Omega_{1}=(-1)^{\frac{(\mathfrak{n}-1)(\mathfrak{n}-2)}{2}}$, we have

$$
\operatorname{det} A_{n}=\frac{\mathfrak{F}_{n}^{n}+\left(1+\mathfrak{F}_{n+1}\right)^{n}}{1-\mathfrak{F}_{n-1}+\mathfrak{F}_{n+1}}
$$

Theorem 3.2. Let $A_{n}=\operatorname{SCirc}\left(\mathfrak{F}_{1}, \ldots, \mathfrak{F}_{n}\right)$ be a matrix as in (1.3) for every positive integer $n$. Then $A_{n}$ is invertible.

Proof. In Theorem 3.1, $\operatorname{det} A_{1}=1 \neq 0$, hence $A_{1}$ is invertible. For $n>1$, according to [13], we have the eigenvalues of $A_{n}$

$$
f\left(\omega^{k} \eta\right)=\sum_{j=1}^{n} \mathfrak{F}_{j}\left(\omega^{k} \eta\right)^{j-1}, \quad(k=1,2, \ldots, n-1),
$$

where $\omega=\exp \left(\frac{2 \pi i}{n}\right), \eta=\exp \left(\frac{\pi i}{n}\right)$.

Note that $\mathfrak{F}_{n}=\frac{\alpha^{n}-\beta^{n}}{\alpha-\beta}$, where $\alpha+\beta=3, \alpha \beta=1$, we get

$$
f\left(\omega^{k} \eta\right)=\frac{1}{\alpha-\beta} \sum_{j=1}^{n}\left(\alpha^{j}-\beta^{j}\right)\left(\omega^{k} \eta\right)^{j-1}, \quad(k=1,2, \ldots, n-1) .
$$

Since $\left|\omega^{k} \eta\right|=1$, and $|\alpha| \neq 1,|\beta| \neq 1$, we have $\omega^{k} \eta \neq \alpha$ and $\omega^{k} \eta \neq \beta$, and hence,

$$
\begin{aligned}
f\left(\omega^{k} \eta\right) & =\frac{\alpha}{\alpha-\beta} \cdot \frac{1+\alpha^{n}}{1-\alpha \omega^{k} \eta}-\frac{\beta}{\alpha-\beta} \cdot \frac{1+\alpha^{n}}{1-\alpha \omega^{k} \eta} \\
& =\frac{\alpha-\beta+\alpha^{n+1}-\beta^{n+1}-\alpha \beta \omega^{k} \eta\left(\alpha^{n}-\beta^{n}\right)}{(\alpha-\beta)\left(1-\alpha \omega^{k} \eta\right)\left(1-\beta \omega^{k} \eta\right)}=\frac{1+\mathfrak{F}_{n+1}-\mathfrak{F}_{n} \omega^{k} \eta}{1-3 \omega^{k} \eta+\omega^{2 k} \eta^{2}}, \quad(k=1,2, \ldots, n-1) .
\end{aligned}
$$

Assume to the contrary that there exists $\omega^{l} \eta(l=1,2, \ldots, n-1)$ such that $f\left(\omega^{l} \eta\right)=0$. We obtain $1+\mathfrak{F}_{n+1}-\mathfrak{F}_{n} \omega^{l} \eta=0$, and hence it follows that $\omega^{l} \eta=\frac{1+\mathfrak{F}_{n+1}}{\mathfrak{F}_{n}}$ is a real number. That is to say, the imaginary part of $\omega^{l} \eta$ is $\sin \frac{(2 l+1) \pi}{n}=0$. Therefore, $\omega^{l} \eta= \pm 1$, and $1+\mathfrak{F}_{n+1}-\mathfrak{F}_{n} \omega^{l} \eta \neq 0(n>0)$. Consequently, we obtain $f\left(\omega^{k} \eta\right) \neq 0$ for any $\omega^{k} \eta(k=1,2, \ldots, n-1)$.

Theorem 3.3. Let $\mathrm{A}_{\mathrm{n}}=\operatorname{SCirc}\left(\mathfrak{F}_{1}, \ldots, \mathfrak{F}_{n}\right)$ be a matrix as in (1.3) for a positive integer $\mathrm{n}>1$. Then

$$
A_{n}^{-1}=\operatorname{SCirc}\left(y_{1}, y_{2}, \ldots, y_{n}\right),
$$


where

$$
\begin{aligned}
& y_{1}=\frac{\left(\mathfrak{F}_{n-1}-1\right) \mathfrak{F}_{n}^{n-2}+\left(1+\mathfrak{F}_{n+1}\right)^{n-1}}{\mathfrak{F}_{\mathfrak{n}}^{n}+\left(1+\mathfrak{F}_{\mathfrak{n}+1}\right)^{\mathfrak{n}}}, \\
& y_{2}=-\frac{\mathfrak{F}_{n}^{n-1}+\left(3+\mathfrak{F}_{\mathfrak{n}+2}\right)\left(1+\mathfrak{F}_{\mathfrak{n}+1}\right)^{\mathfrak{n}-2}}{\mathfrak{F}_{\mathfrak{n}}^{n}+\left(1+\mathfrak{F}_{\mathfrak{n}+1}\right)^{\mathrm{n}}}, \\
& y_{k}=\frac{\left(1-\mathfrak{F}_{n-1}+\mathfrak{F}_{n+1}\right) \mathfrak{F}_{n}^{k-3}\left(1+\mathfrak{F}_{n+1}\right)^{n-k}}{\mathfrak{F}_{\mathfrak{n}}^{n}+\left(1+\mathfrak{F}_{n+1}\right)^{n}}, \quad(k=3,4, \ldots, n) .
\end{aligned}
$$

Proof. Let

$$
\Omega_{2}=\left(\begin{array}{cccccc}
1 & -\frac{l_{n}^{\prime}}{\mathfrak{F}_{1}} & \omega_{3} & \omega_{4} & \cdots & \omega_{n} \\
0 & 1 & -\frac{\mathfrak{F}_{n-2}}{l_{n}} & -\frac{\mathfrak{F}_{n-3}}{l_{n}} & \cdots & -\frac{\mathfrak{F}_{1}}{l_{n}} \\
\vdots & \ddots & 1 & 0 & \cdots & 0 \\
\vdots & & \ddots & \ddots & \ddots & \vdots \\
\vdots & & & \ddots & \ddots & 0 \\
0 & \cdots & \cdots & \cdots & 0 & 1
\end{array}\right)_{n \times n}
$$

where

$$
\omega_{j}=\frac{l_{n}^{\prime}}{l_{n}} \mathfrak{F}_{n+1-j}-\mathfrak{F}_{\mathfrak{n}+2-j}, \quad(j=3,4, \ldots, n),
$$

$l_{n}^{\prime}$ and $l_{n}$ are respectively given by (3.3) and (3.4). Multiplying (3.2) by $\Omega_{2}$ from right,

$$
\Sigma A_{n} \Omega_{1} \Omega_{2}=\left(\begin{array}{ccccccc}
\mathfrak{F}_{1} & 0 & \ldots & \cdots & \cdots & \cdots & 0 \\
0 & l_{n} & \ddots & & & & \vdots \\
\vdots & 0 & \mathfrak{F}_{1}+\mathfrak{F}_{n+1} & \ddots & & & \vdots \\
\vdots & \vdots & -\mathfrak{F}_{n} & \ddots & \ddots & & \vdots \\
\vdots & \vdots & 0 & \ddots & \ddots & \ddots & \vdots \\
\vdots & \vdots & \vdots & \ddots & \ddots & \ddots & 0 \\
0 & 0 & 0 & \cdots & 0 & -\mathfrak{F}_{n} & \mathfrak{F}_{1}+\mathfrak{F}_{n+1}
\end{array}\right)_{n \times n}
$$

where $\Sigma$ and $\Omega_{1}$ are the same as Theorem 3.1.

Let $\Sigma A_{n} \Omega_{1} \Omega_{2}=\mathcal{D} \oplus \mathcal{H}$, where $\mathrm{D}=\operatorname{diag}\left(1, l_{n}\right)$ is a diagonal matrix, $\mathcal{H}$ is as defined in Lemma 2.2, and $\mathcal{D} \oplus \mathcal{H}$ is the direct sum of $\mathcal{D}$ and $\mathcal{H}$. Denote $\Omega=\Omega_{1} \Omega_{2}$, then we obtain $A_{n}^{-1}=\Omega\left(\mathcal{D}^{-1} \oplus \mathcal{H}^{-1}\right) \Sigma$.

Since the last row elements of the matrix $\Omega$ are $\left(0,1,-\frac{\widetilde{F}_{n-2}}{l_{n}},-\frac{\widetilde{\mathfrak{F}}_{\mathfrak{n}-3}}{l_{n}}, \ldots,-\frac{\widetilde{\mathfrak{F}}_{2}}{l_{n}},-\frac{\widetilde{\mathfrak{F}}_{1}}{l_{n}}\right)$, the last row elements of the matrix $\Omega\left(\mathcal{D}^{-1} \oplus \mathcal{H}^{-1}\right)$ are $\left(0, \frac{1}{l_{n}}, T_{3}, T_{4}, \ldots, T_{n}\right)$, where

$$
\mathrm{T}_{\mathrm{k}}=\sum_{i=1}^{\mathrm{n}+1-\mathrm{k}}-\frac{\mathfrak{F}_{n+2-k-i} \mathfrak{F}_{n}^{i-1}}{l_{n}\left(1+\mathfrak{F}_{n+1}\right)^{i}}, \quad(k=3,4, \ldots, n) .
$$

Using (2.4), we simplify the above equation as

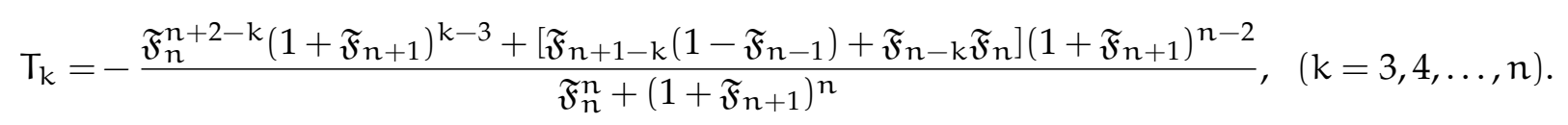

The last row of $A_{n}^{-1}=\operatorname{SCirc}\left(y_{1}, y_{2}, \ldots, y_{n}\right)$ is $\left(-y_{2},-y_{3}, \ldots,-y_{n}, y_{1}\right)$, which is given by the following equations:

$$
-y_{2}=\frac{3}{l_{n}}-T_{3}
$$




$$
\begin{aligned}
-\mathrm{y}_{3} & =\mathrm{T}_{\mathrm{n}}, \\
-\mathrm{y}_{4} & =\mathrm{T}_{\mathrm{n}-1}-3 \mathrm{~T}_{\mathrm{n}}, \\
-\mathrm{y}_{\mathrm{k}} & =\mathrm{T}_{\mathrm{n}-\mathrm{k}+3}-3 \mathrm{~T}_{\mathrm{n}-\mathrm{k}+4}+\mathrm{T}_{\mathrm{n}-\mathrm{k}+5}, \quad \mathrm{k}=5,6, \ldots, \mathrm{n}, \\
\mathrm{y}_{1} & =\frac{1}{l_{\mathrm{n}}}-3 \mathrm{~T}_{3}+\mathrm{T}_{4} .
\end{aligned}
$$

Applying (3.9) into above equations, we compute

$$
\begin{aligned}
y_{1} & =\frac{\left(\mathfrak{F}_{n-1}-1\right) \mathfrak{F}_{n}^{n-2}+\left(1+\mathfrak{F}_{n+1}\right)^{n-1}}{\mathfrak{F}_{\mathfrak{n}}^{n}+\left(1+\mathfrak{F}_{n+1}\right)^{n}}, \\
y_{2} & =-\frac{\mathfrak{F}_{n}^{n-1}+\left(3+\mathfrak{F}_{n+2}\right)\left(1+\mathfrak{F}_{n+1}\right)^{n-2}}{\mathfrak{F}_{n}^{n}+\left(1+\mathfrak{F}_{n+1}\right)^{n}}, \\
y_{k} & =\frac{\left(1-\mathfrak{F}_{n-1}+\mathfrak{F}_{n+1}\right) \mathfrak{F}_{n}^{k-3}\left(1+\mathfrak{F}_{n+1}\right)^{n-k}}{\mathfrak{F}_{n}^{n}+\left(1+\mathfrak{F}_{n+1}\right)^{n}}, \quad(k=3,4, \ldots, n) .
\end{aligned}
$$

3.2. Norms and spread of skew circulant matrix involving the product of Fibonacci and Lucas numbers

In this subsection, we further investigate the properties of skew circulant matrices involving the product of Fibonacci and Lucas numbers, such as their norms, lower and upper bounds for their spread.

Theorem 3.4. Let $A_{\mathfrak{n}}=\operatorname{SCirc}\left(\mathfrak{F}_{1}, \ldots, \mathfrak{F}_{\mathfrak{n}}\right)$ be a matrix as in (1.3). Then the maximum column sum matrix norm, the maximum row sum matrix norm and the Frobenius norm of $A_{n}$ are given by

$$
\begin{aligned}
\left\|A_{n}\right\|_{1} & =\left\|A_{n}\right\|_{\infty}=2 \mathfrak{F}_{n}-\mathfrak{F}_{n-1}-1, \\
\left\|A_{n}\right\|_{F} & =\sqrt{\frac{n\left(\mathfrak{F}_{\mathfrak{n}+1}^{2}-\mathfrak{F}_{\mathfrak{n}}^{2}-8 \mathfrak{n}-7\right)}{5}},
\end{aligned}
$$

where $\mathfrak{F}_{n}$ is the $\mathrm{nth}$ product of Fibonacci and Lucas numbers.

Proof. According to the Definition 4 in [18], (2.1), and (2.3), we have

$$
\begin{aligned}
& \left\|A_{n}\right\|_{1}=\sum_{i=1}^{n}\left|\mathfrak{F}_{\mathfrak{i}}\right|=2 \mathfrak{F}_{n}-\mathfrak{F}_{n-1}-1, \\
& \left\|A_{n}\right\|_{\infty}=\sum_{i=1}^{n}\left|\mathfrak{F}_{\mathfrak{i}}\right|=2 \mathfrak{F}_{n}-\mathfrak{F}_{n-1}-1, \\
& \left\|A_{n}\right\|_{F}=\sqrt{n \sum_{i=1}^{n}\left|\mathfrak{F}_{\mathfrak{i}}\right|^{2}}=\sqrt{\frac{n\left(\mathfrak{F}_{n+1}^{2}-\mathfrak{F}_{n}^{2}-8 n-7\right)}{5}} .
\end{aligned}
$$

Theorem 3.5. Let $A_{n}^{\prime}=\operatorname{SCirc}\left(\mathfrak{F}_{1},-\mathfrak{F}_{2}, \ldots,-\mathfrak{F}_{n-1}, \mathfrak{F}_{n}\right)$ be an odd-order alternative skew circulant matrix. Then

$$
\left\|A_{n}^{\prime}\right\|_{2}=2 \mathfrak{F}_{n}-\mathfrak{F}_{n-1}-1,
$$

where $\mathfrak{F}_{n}$ is the $\mathrm{n}^{\text {th }}$ product of Fibonacci and Lucas numbers.

Proof. In [13], we have the eigenvalues of $A_{n}^{\prime}$

$$
\lambda_{j}\left(A_{n}^{\prime}\right)=\sum_{i=1}^{n}(-1)^{i-1} \mathfrak{F}_{i}\left(\omega^{j} \eta\right)^{i-1}, \quad(j=0,1, \cdots, n-1) .
$$


Using the absolute value inequality,

$$
\left|\lambda_{j}\left(A_{n}^{\prime}\right)\right| \leqslant \sum_{i=1}^{n}\left|(-1)^{i-1} \mathfrak{F}_{i}\right| \cdot\left|\left(\omega^{j} \eta\right)^{i-1}\right|=\sum_{i=1}^{n} \mathfrak{F}_{i}, \quad(j=0,1, \ldots, n-1) .
$$

Since $n$ is odd, $\sum_{i=1}^{n} \mathfrak{F}_{i}$ is an eigenvalue of $A_{n}^{\prime}$, which is

$$
\left(\begin{array}{ccccc}
\mathfrak{F}_{1} & -\mathfrak{F}_{2} & \mathfrak{F}_{3} & \cdots & \mathfrak{F}_{\mathfrak{n}} \\
-\mathfrak{F}_{n} & \ddots & \ddots & \ddots & \vdots \\
\mathfrak{F}_{n-1} & \ddots & \ddots & \ddots & \mathfrak{F}_{3} \\
\vdots & \ddots & \ddots & \ddots & -\mathfrak{F}_{2} \\
\mathfrak{F}_{2} & \cdots & \mathfrak{F}_{\mathfrak{n}-1} & -\mathfrak{F}_{\mathfrak{n}} & \mathfrak{F}_{1}
\end{array}\right)_{\mathrm{n} \times \mathrm{n}}\left(\begin{array}{c}
1 \\
-1 \\
1 \\
-1 \\
\vdots \\
1
\end{array}\right)_{\mathrm{n} \times 1}=\sum_{i=1}^{n} \mathfrak{F}_{i} \cdot\left(\begin{array}{c}
1 \\
-1 \\
1 \\
-1 \\
\vdots \\
1
\end{array}\right) .
$$

To sum up, we obtain

$$
\max _{0 \leqslant j \leqslant n-1}\left|\lambda_{j}\left(A_{n}^{\prime}\right)\right|=\sum_{i=1}^{n} \mathfrak{F}_{i}
$$

Since skew circulant matrices $A_{n}^{\prime}$ satisfies $\left(A_{n}^{\prime}\right)^{\mathrm{H}} A_{n}^{\prime}=A_{n}^{\prime}\left(A_{n}^{\prime}\right)^{\mathrm{H}}\left(\left(A_{n}^{\prime}\right)^{\mathrm{H}}\right.$ is the conjugate transpose of $\left.A_{n}^{\prime}\right)$, skew circulant matrices are normal. By Lemma 7 in [18], (2.1), and (3.12), we have

$$
\left\|A_{n}^{\prime}\right\|_{2}=\sum_{i=1}^{n} \mathfrak{F}_{i}=2 \mathfrak{F}_{n}-\mathfrak{F}_{n-1}-1
$$

Theorem 3.6. Let $A_{n}=\operatorname{SCirc}\left(\mathfrak{F}_{1}, \ldots, \mathfrak{F}_{n}\right)$ be a matrix as in (1.3). Then a lower bound and an upper bound for the spread $s\left(A_{n}\right)$ of $A_{n}$ are given as follows:

$$
\frac{\left|2-2 n+(n+4) \mathfrak{F}_{n}-(n+2) \mathfrak{F}_{n+1}\right|}{n-1} \leqslant s\left(A_{n}\right) \leqslant \sqrt{\frac{2 n\left(\mathfrak{F}_{n+1}^{2}-\mathfrak{F}_{n}^{2}-8 n-7\right)}{5}-2 n,}
$$

where $\mathfrak{F}_{\mathrm{n}}$ is the $\mathrm{n}^{\text {th }}$ product of Fibonacci and Lucas numbers.

Proof. Note that the trace of $A_{n}$ is $\operatorname{tr} A_{n}=n \mathfrak{F}_{1}=n$. The sum of the off-diagonal of $A_{n}$ is

$$
\ell\left(A_{n}\right)=\sum_{k=2}^{n}(n-k+1) \mathfrak{F}_{k}-\sum_{k=2}^{n}(k-1) \mathfrak{F}_{k}=(n+2) \sum_{k=2}^{n} \mathfrak{F}_{k}-2 \sum_{k=2}^{n} k \mathfrak{F}_{k} .
$$

By (2.1) and (2.2), we have

$$
\ell\left(A_{n}\right)=2-2 n+(n+4) \mathfrak{F}_{n}-(n+2) \mathfrak{F}_{n+1} .
$$

Since skew circulant matrix $A_{n}$ satisfies $A_{n}^{H} A_{n}=A_{n} A_{n}^{H}\left(A_{n}^{H}\right.$ is the conjugate transpose of $\left.A_{n}\right)$, skew circulant matrix is normal. Since $A_{n}$ is a normal and real matrix, by Definition 5 and Lemma 6 in [18] and (3.11), we get

$$
\frac{\left|2-2 n+(n+4) \mathfrak{F}_{n}-(n+2) \mathfrak{F}_{n+1}\right|}{n-1} \leqslant s\left(A_{n}\right) \leqslant \sqrt{\frac{2 n\left(\mathfrak{F}_{n+1}^{2}-\mathfrak{F}_{n}^{2}-8 n-7\right)}{5}-2 n .}
$$


4. Determinant, inverse, norms and spread of skew left circulant matrix involving the product of Fibonacci and Lucas numbers

In this section, let $A_{n}^{\prime \prime}=\operatorname{SLCirc}\left(\mathfrak{F}_{1}, \ldots, \mathfrak{F}_{n}\right)$ be a skew left circulant matrix. According to Lemma 2.3, we know that skew circulant matrix and skew left circulant matrix can be transformed into each other. Therefore, using the obtained conclusions in Section 3.1, we get a determinant explicit formula for $A_{n}^{\prime \prime}$. Besides, we prove that $A_{n}^{\prime \prime}$ is invertible for every positive integer $n$ and compute the inverse of $A_{n}^{\prime \prime}$. We also study the properties of skew left circulant matrices involving the product of Fibonacci and Lucas numbers.

\subsection{Determinant and inverse of skew left circulant matrix involving the product of Fibonacci and Lucas numbers}

In this subsection, we get a determinant explicit formula for $A_{n}^{\prime \prime}$ in Theorem 4.1. Besides, we prove that $A_{n}^{\prime \prime}$ is invertible for every positive integer $n$ and compute the inverse of $A_{n}^{\prime \prime}$ in Theorems 4.2 and 4.3, respectively.

Theorem 4.1. Let $A_{n}^{\prime \prime}=\operatorname{SLCirc}\left(\mathfrak{F}_{1}, \ldots, \mathfrak{F}_{n}\right)$ be a matrix as in (1.4) for a positive integer $n$. Then

$$
\operatorname{det} A_{\mathfrak{n}}^{\prime \prime}=(-1)^{\frac{\mathfrak{n}(\mathfrak{n}-1)}{2}} \frac{\mathfrak{F}_{n}^{n}+\left(1+\mathfrak{F}_{\mathfrak{n}+1}\right)^{n}}{1-\mathfrak{F}_{\mathfrak{n}-1}+\mathfrak{F}_{\mathfrak{n}+1}}
$$

where $\mathfrak{F}_{\mathrm{n}}$ is the $\mathrm{n}^{\text {th }}$ product of Fibonacci and Lucas numbers.

Theorem 4.2. Let $A_{n}^{\prime \prime}=\operatorname{SLCirc}\left(\mathfrak{F}_{1}, \ldots, \mathfrak{F}_{\mathfrak{n}}\right)$ be a matrix as in (1.4) for every positive interger $n$. Then $A_{n}^{\prime \prime}$ is invertible.

Theorem 4.3. Let $\mathrm{A}_{\mathfrak{n}}^{\prime \prime}=\operatorname{SLCirc}\left(\mathfrak{F}_{1}, \ldots, \mathfrak{F}_{\mathfrak{n}}\right)$ be a matrix as in $(1.4)$ for a positive integer $\mathrm{n}>1$. Then

$$
\left(A^{\prime \prime} n\right)^{-1}=\operatorname{SLCirc}\left(y_{1}^{\prime \prime}, y_{2}^{\prime \prime}, \ldots, y_{n}^{\prime \prime}\right)
$$

where

$$
\begin{aligned}
& y_{1}^{\prime \prime}=\frac{\left(\mathfrak{F}_{\mathfrak{n}-1}-1\right) \mathfrak{F}_{\mathfrak{n}}^{\mathfrak{n}-2}+\left(1+\mathfrak{F}_{\mathfrak{n}+1}\right)^{\mathfrak{n}-1}}{\mathfrak{F}_{\mathfrak{n}}^{\mathfrak{n}}+\left(1+\mathfrak{F}_{\mathfrak{n}+1}\right)^{\mathrm{n}}} \\
& y_{k}^{\prime \prime}=-\frac{\mathfrak{F}_{\mathfrak{n}}^{n-1-k}\left(1+\mathfrak{F}_{\mathfrak{n}+1}\right)^{k-2}\left[\mathfrak{F}_{\mathfrak{n}}^{2}+\left(1-\mathfrak{F}_{\mathfrak{n}-1}\right)\left(1+\mathfrak{F}_{\mathfrak{n}+1}\right)\right]}{\mathfrak{F}_{\mathfrak{n}}^{n}+\left(1+\mathfrak{F}_{\mathfrak{n}+1}\right)^{n}}, \quad(k=2,3, \ldots, n-1), \\
& y_{n}^{\prime \prime}=\frac{\mathfrak{F}_{n}^{n-1}+\left(3+\mathfrak{F}_{n+2}\right)\left(1+\mathfrak{F}_{\mathfrak{n}+1}\right)^{\mathfrak{n}-2}}{\mathfrak{F}_{n}^{n}+\left(1+\mathfrak{F}_{\mathfrak{n}+1}\right)^{\mathfrak{n}}} \text {. }
\end{aligned}
$$

4.2. Norms and spread of skew left circulant matrix involving the product of Fibonacci and Lucas numbers

Like Section 3.2, we study some properties of skew left circulant matrices involving the product of Fibonacci and Lucas numbers.

Theorem 4.4. Let $A_{n}^{\prime \prime}=\operatorname{SLCirc}\left(\mathfrak{F}_{1}, \ldots, \mathfrak{F}_{n}\right)$ be a matrix as in (1.4). Then three kinds norms of $A_{n}^{\prime \prime}$ are given by

$$
\begin{aligned}
\left\|A_{n}^{\prime \prime}\right\|_{1} & =\left\|A_{n}^{\prime \prime}\right\|_{\infty}=2 \mathfrak{F}_{n}-\mathfrak{F}_{n-1}-1, \\
\left\|A_{n}^{\prime \prime}\right\|_{F} & =\sqrt{\frac{n\left(\mathfrak{F}_{n+1}^{2}-\mathfrak{F}_{n}^{2}-8 n-7\right)}{5}},
\end{aligned}
$$

where $\mathfrak{F}_{\mathrm{n}}$ is the $\mathrm{n}^{\text {th }}$ product of Fibonacci and Lucas numbers.

Proof. Using the similar method in Theorem 3.4, the conclusion is obtained. 
Theorem 4.5. Let $A_{n}^{\prime \prime \prime}=\operatorname{SLCirc}\left(\mathfrak{F}_{1},-\mathfrak{F}_{2}, \ldots,-\mathfrak{F}_{n-1}, \mathfrak{F}_{n}\right)$ be an odd-order alternative skew left circulant matrix. Then

$$
\left\|A_{n}^{\prime \prime \prime}\right\|_{2}=2 \mathfrak{F}_{n}-\mathfrak{F}_{n-1}-1,
$$

where $\mathfrak{F}_{\mathrm{n}}$ is the $\mathrm{n}^{\text {th }}$ product of Fibonacci and Lucas numbers.

Proof. By Lemma 2.4, we have

$$
\lambda_{j}\left(A_{n}^{\prime \prime \prime}\right)= \pm\left|\sum_{i=1}^{n}(-1)^{i-1} \mathfrak{F}_{i} \omega^{\left(j-\frac{1}{2}\right)(k-1)}\right|, \quad\left(j=1,2, \ldots, \frac{n-1}{2}\right), \quad \lambda_{\frac{n+1}{2}}\left(A_{n}^{\prime \prime \prime}\right)=\sum_{i=1}^{n} \mathfrak{F}_{i} .
$$

Therefore,

$$
\left|\lambda_{j}\left(A_{n}^{\prime \prime \prime}\right)\right| \leqslant \sum_{i=1}^{n}\left|(-1)^{i-1} \mathfrak{F}_{\mathfrak{i}}(-1)^{i-1}\right|=\sum_{i=1}^{n} \mathfrak{F}_{i}, \quad\left(j=1,2, \ldots, \frac{n-1}{2}\right) .
$$

By (4.8) and (4.9), we gain

$$
\max _{0 \leqslant i \leqslant n+1}\left|\lambda_{i}\left(A_{n}^{\prime \prime \prime}\right)\right|=\sum_{i=1}^{n} \mathfrak{F}_{i}
$$

Since skew left circulant matrix $A_{n}^{\prime \prime \prime}$ satisfies $\left(A_{n}^{\prime \prime \prime}\right)^{\mathrm{H}} A_{n}^{\prime \prime \prime}=A_{n}^{\prime \prime \prime}\left(A_{n}^{\prime \prime \prime}\right)^{\mathrm{H}}\left(\left(A_{n}^{\prime \prime \prime}\right)^{\mathrm{H}}\right.$ is the conjugate transpose of $\left.A_{n}^{\prime \prime \prime}\right)$, skew circulant matrix is normal. By Lemma 7 in [18], (2.1), and (4.10), we obtain

$$
\left\|A_{n}^{\prime \prime \prime}\right\|_{2}=2 \mathfrak{F}_{n}-\mathfrak{F}_{n-1}-1 .
$$

Theorem 4.6. Suppose that $\mathrm{A}_{n}^{\prime \prime}=\operatorname{SLCirc}\left(\mathfrak{F}_{1}, \ldots, \mathfrak{F}_{n}\right)$ be a matrix as in (1.4). The lower and upper bounds for the spread of $A_{n}^{\prime \prime}$ are

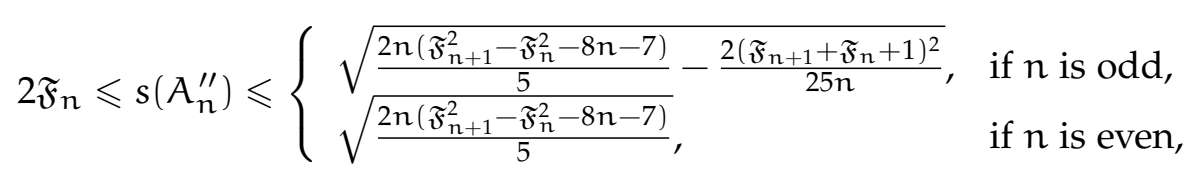

where $\mathfrak{F}_{n}$ is the $\mathrm{n}^{\text {th }}$ product of Fibonacci and Lucas numbers.

Proof. Since $A_{n}^{\prime \prime}$ is a symmetric matrix, by Definition 5 and Lemma 6 in [18],

$$
2 \max \left|A_{i}^{\prime \prime}\right| \leqslant s\left(A_{n}^{\prime \prime}\right) \leqslant \sqrt{2\left\|A_{n}^{\prime \prime}\right\|_{F}^{2}-\frac{2}{n}\left|\operatorname{tr} A_{n}^{\prime \prime}\right|^{2}}
$$

For $A_{n}^{\prime \prime}$,

$$
2 \max \left|A_{i}^{\prime \prime}\right|=2 \mathfrak{F}_{n} .
$$

On the other hand, according to Theorem 4.4,

$$
\left\|A_{n}^{\prime \prime}\right\|_{F}=\sqrt{\frac{n\left(\mathfrak{F}_{\mathfrak{n}+1}^{2}-\mathfrak{F}_{\mathfrak{n}}^{2}-8 n-7\right)}{5}} .
$$

If $n$ is odd, we have

$$
\operatorname{tr}\left(A_{n}^{\prime \prime}\right)=\mathfrak{F}_{1}-\mathfrak{F}_{2}+\mathfrak{F}_{3}-\cdots+\mathfrak{F}_{n}=\sum_{i=0}^{\frac{n-1}{2}} \mathfrak{F}_{2 i+1}-\sum_{i=1}^{\frac{n-1}{2}} \mathfrak{F}_{2 i} .
$$

Note that

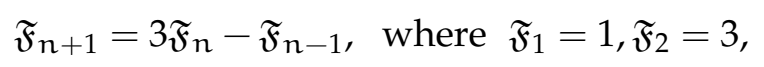


we conclude that

$$
\begin{aligned}
\sum_{i=0}^{\frac{n-1}{2}} \mathfrak{F}_{2 i+1}- & \sum_{i=1}^{\frac{n-1}{2}} \mathfrak{F}_{2 i}=2 \sum_{i=1}^{\frac{n-1}{2}} \mathfrak{F}_{2 i}-\sum_{i=0}^{\frac{n-1}{2}} \mathfrak{F}_{2 i+1}+\mathfrak{F}_{1}+\mathfrak{F}_{n} \\
\sum_{i=1}^{\frac{n-1}{2}} \mathfrak{F}_{2 i} & =3 \sum_{i=0}^{\frac{n-1}{2}} \mathfrak{F}_{2 i+1}-3 \mathfrak{F}_{1}-3 \mathfrak{F}_{n}-\sum_{i=1}^{\frac{n-1}{2}} \mathfrak{F}_{2 i}+\mathfrak{F}_{2}+\mathfrak{F}_{n-1}
\end{aligned}
$$

Hence

$$
\operatorname{tr}\left(A_{n}^{\prime \prime}\right)=\frac{1}{5}\left(\mathfrak{F}_{n+1}+\mathfrak{F}_{n}+1\right)
$$

If $n$ is even, then

$$
\operatorname{tr}\left(A_{n}^{\prime \prime}\right)=\mathfrak{F}_{1}-\mathfrak{F}_{1}+\mathfrak{F}_{3}-\mathfrak{F}_{3}+\cdots-\mathfrak{F}_{\mathfrak{n}-1}=0 .
$$

So the result is as follows:

$$
2 \mathfrak{F}_{n} \leqslant s\left(A_{n}^{\prime \prime}\right) \leqslant \begin{cases}\sqrt{\frac{2 n\left(\mathfrak{F}_{n+1}^{2}-\mathfrak{F}_{n}^{2}-8 \mathfrak{n}-7\right)}{5}-\frac{2\left(\mathfrak{F}_{n+1}+\mathfrak{F}_{n}+1\right)^{2}}{25 n}}, & \text { if } n \text { is odd } \\ \sqrt{\frac{2 n\left(\mathfrak{F}_{n+1}^{2}-\mathfrak{F}_{n}^{2}-8 n-7\right)}{5}}, & \text { if } n \text { is even. }\end{cases}
$$

\section{Algorithms and numerical examples}

In this section, we give two algorithms for computing inverses of skew circulant and skew left circulant matrices involving the product of Fibonacci and Lucas numbers. Then, several examples are given to show the correctness of these algorithms. Besides, we compute determinants, three kinds of norms and lower $\&$ upper bounds for spread of these matrices.

Based on Theorem 3.1, given the skew circulant matrix involving the product of Fibonacci and Lucas numbers $A_{n}=\operatorname{SCirc}\left(\mathfrak{F}_{1}, \ldots, \mathfrak{F}_{n}\right)$, the algorithm for finding its inverse is as follows.

\section{Algorithm 5.1.}

Step 1: Input $\mathrm{n}$ and generate the product of Fibonacci and Lucas numbers by (1.2).

Step 2: Compute $y_{i}(i=1,2, \cdots, n)$ via (3.6)-(3.8).

Step 3: Output $A_{n}^{-1}=\operatorname{SCirc}\left(y_{1}, y_{2}, \ldots, y_{n}\right)$ by (3.5).

Example 5.2. Consider a $5 \times 5$ skew circulant matrix involving the product of Fibonacci and Lucas numbers $A_{5}$

$$
A_{5}=\left[\begin{array}{ccccc}
1 & 3 & 8 & 21 & 55 \\
-55 & 1 & 3 & 8 & 21 \\
-21 & -55 & 1 & 3 & 8 \\
8 & -21 & -55 & 1 & 3 \\
3 & 8 & -21 & -55 & 1
\end{array}\right]
$$

Above all, according to (3.1), $\operatorname{det} A_{5}=516805000$. In particular, by (3.10) and (3.11), the three kinds norms of $A_{5}$ are given by

$$
\left\|A_{5}\right\|_{1}=\left\|A_{5}\right\|_{\infty}=88, \quad\left\|A_{5}\right\|_{F}=\sqrt{17664} .
$$

By (3.13), the lower and upper bounds for spread of $A_{5}$ are

$$
\frac{521}{4} \leqslant s\left(A_{5}\right) \leqslant \sqrt{35318}
$$


As for $A_{5}^{-1}$, according to Algorithm 5.1, we have

$$
y_{1}=\frac{142521}{20672200}, \quad y_{2}=-\frac{373641}{20672200}, \quad y_{3}=\frac{841}{20672200}, \quad y_{4}=\frac{319}{20672200}, \quad y_{5}=\frac{121}{20672200}
$$

Finally,

$$
\left(A_{5}\right)^{-1}=\left[\begin{array}{ccccc}
\frac{142521}{20672200} & -\frac{373641}{20672200} & \frac{841}{20672200} & \frac{319}{20672200} & \frac{121}{20672200} \\
-\frac{121}{20672200} & \frac{142521}{20672200} & -\frac{373641}{20672200} & \frac{841}{20672200} & \frac{319}{20672200} \\
-\frac{319}{20672200} & -\frac{121}{20672200} & \frac{142521}{20672200} & -\frac{373641}{20672200} & \frac{841}{20672200} \\
-\frac{841}{20672200} & -\frac{319}{20672200} & -\frac{121}{20672200} & \frac{142521}{20672200} & -\frac{373641}{20672200} \\
\frac{373641}{20672200} & -\frac{841}{20672200} & -\frac{319}{20672200} & -\frac{121}{20672200} & \frac{142521}{20672200}
\end{array}\right] .
$$

Meanwhile, based on Theorem 4.3, given a skew left circulant matrix involving the product of Fibonacci and Lucas numbers $A_{n}^{\prime \prime}=\operatorname{SLCirc}\left(\mathfrak{F}_{1}, \ldots, \mathfrak{F}_{n}\right)$, the algorithm for finding its inverse is as follows.

\section{Algorithm 5.3.}

Step 1: Input $n$ and generate the product of Fibonacci and Lucas numbers by (1.2).

Step 2: Compute $y_{i}^{\prime \prime}(i=1,2, \cdots, n)$ via (4.3)-(4.5).

Step 3: Output $A_{n}^{\prime \prime-1}=\operatorname{SLCirc}\left(y_{1}^{\prime \prime}, y_{2}^{\prime \prime}, \ldots, y_{n}^{\prime \prime}\right)$ by (4.2).

Example 5.4. Consider a $5 \times 5$ skew left circulant matrix involving the product of Fibonacci and Lucas numbers $A_{5}^{\prime \prime}$ :

$$
A_{5}^{\prime \prime}=\left[\begin{array}{ccccc}
1 & 3 & 8 & 21 & 55 \\
3 & 8 & 21 & 55 & -1 \\
8 & 21 & 55 & -1 & -3 \\
21 & 55 & -1 & -3 & -8 \\
55 & -1 & -3 & -8 & -21
\end{array}\right]
$$

Firstly, we get $\operatorname{det} A_{5}^{\prime \prime}=516805000$ from (4.1) and the three kinds norms of $A_{5}^{\prime \prime}$ by (4.6) and (4.7)

$$
\left\|A_{5}^{\prime \prime}\right\|_{1}=\left\|A_{5}^{\prime \prime}\right\|_{\infty}=88, \quad\left\|A_{5}^{\prime \prime}\right\|_{\mathrm{F}}=\sqrt{17664} .
$$

In Theorem 4.6, the lower and upper bounds for spread of $A_{5}^{\prime \prime}$ are

$$
110 \leqslant s\left(A_{5}^{\prime \prime}\right) \leqslant \sqrt{34688}
$$

As for $A^{\prime \prime}{ }_{5}^{-1}$, according to Algorithm 5.3, compute

$$
y_{1}^{\prime \prime}=\frac{142521}{20672200}, \quad y_{2}^{\prime \prime}=-\frac{121}{20672200}, \quad y_{3}^{\prime \prime}=-\frac{319}{20672200}, \quad y_{4}^{\prime \prime}=-\frac{841}{20672200}, \quad y_{5}^{\prime \prime}=\frac{373641}{20672200}
$$

Finally,

$$
\left(A_{5}^{\prime \prime}\right)^{-1}=\left[\begin{array}{ccccc}
\frac{142521}{20672200} & -\frac{121}{20672200} & -\frac{319}{20672200} & -\frac{841}{20672200} & \frac{373641}{20672200} \\
-\frac{121}{20672200} & -\frac{319}{20672200} & -\frac{841}{20672200} & \frac{373641}{20672200} & -\frac{142521}{20672200} \\
-\frac{319}{20672200} & -\frac{841}{20672200} & \frac{373641}{20672200} & -\frac{142521}{20672200} & \frac{121}{20672200} \\
-\frac{841}{20672200} & \frac{373641}{20672200} & -\frac{142521}{20672200} & \frac{121}{20672200} & \frac{319}{20672200} \\
\frac{373641}{20672200} & -\frac{142521}{20672200} & \frac{121}{20672200} & \frac{319}{20672200} & \frac{841}{20672200}
\end{array}\right]
$$




\section{Conclusions}

We discuss the invertibility of the skew circulant and skew left circulant matrices involving the product of Fibonacci and Lucas numbers and present the determinant and inverse matrices by constructing the transformation matrices. The four kinds of norms and bounds for the spread of these matrices are given, respectively. To test the validity of our method, we design two algorithms (Algorithms 5.1 and 5.3), and then conduct two examples.

\section{Acknowledgment}

The research was supported by National Natural Science Foundation of China (Grant No.11671187), Natural Science Foundation of Shandong Province (Grant No.ZR2016AM14), and the PhD Research Foundation of Linyi University (Grant No.LYDX2018BS067).

\section{References}

[1] A. Daher, E. H. Baghious, G. Burel, Fast algorithm for optimal design of block digital filters based on circulant matrices, IEEE Signal Process. Lett., 15 (2008), 637-640. 1

[2] P. J. Davis, Circulant Matrices, John Wiley \& Sons, New York-Chichester-Brisbane, (1979). 1

[3] Q. H. Feng, F. W. Meng, Explicit solutions for space-time fractional partial differential equations in mathematical physics by a new generalized fractional Jacobi elliptic equation-based sub-equation method, Optik, 127 (2016), 7450-7458. 1

[4] D. Q. Fu, Z. L. Jiang, Y. F. Cui, S. T. Jhang, New fast algorithm for optimal design of block digital filters by skew-cyclic convolution, IET Signal Process., 8 (2014), 633-638. 1

[5] Y. Gao, Z. L. Jiang, Y. P. Gong, On the determinants and inverses of skew circulant and skew left circulant matrices with Fibonacci and Lucas Numbers, Wseas Trans. Math., 12 (2013), 472-481. 1, 2.3

[6] R. P. Grimaldi, Fibonacci and Catalan Numbers, John Wiley \& Sons, Hoboken, (2012). 1

[7] C. Hellings, W. Utschick, Block-skew-circulant matrices in complex-valued signal processing, IEEE Trans. Signal Process., 63 (2015), 2093-2107. 1

[8] T. Huckle, Circulant and skewcirculant matrices for solving Toeplitz matrix problems, SIAM J. Matrix Anal. Appl., 13 (1992), 767-777. 1

[9] A. İpek, On the spectral norms of circulant matrices with classical Fibonacci and Lucas numbers entries, Appl. Math. Comput., 217 (2011), 6011-6012. 1

[10] X. Y. Jiang, K. C. Hong, Exact determinants of some special circulant matrices involving four kinds of famous numbers, Abstr. Appl. Anal., 2014 (2014), 12 pages. 1

[11] X. Y. Jiang, K. C. Hong, Explicit inverse matrices of Tribonacci skew circulant type matrices, Appl. Math. Comput., 268 (2015), 93-102. 1

[12] X. Y. Jiang, K. C. Hong, Explicit form of determinants and inverse matrices of Tribonacci r-circulant type matrices, J. Math. Chem., 56 (2018), 1234-1249. 1

[13] Z. L. Jiang, Z. X. Zhou, Circulant Matrices, Chengdu Technology University Publishing Company, Chengdu, (1999). $1,3.1,3.2$

[14] X.-Q. Jin, S.-L. Lei, Y.-M. Wei, Circulant preconditioners for solving singular perturbation delay differential equations, Numer. Linear Algebra Appl., 12 (2005), 327-336. 1

[15] H. Karner, J. Schneid, C. W. Ueberhuber, Spectral decomposition of real circulant matrices, Linear Algebra Appl., 367 (2003), 301-311. 2.4

[16] T. Koshy, Fibonacci and Lucas Numbers with Applications, Wiley-Interscience, New York, (2001). 1

[17] T. Koshy, Pell and Pell-Lucas numbers with applications, Springer, New York, (2014). 1

[18] J. Li, Z. L. Jiang, F. L. Lu, Determinants, norms, and the spread of circulant matrices with Tribonacci and generalized Lucas numbers, Abstr. Appl. Anal., 2014 (2014), 9 pages. 1, 3.2, 3.2, 3.2, 4.2, 4.2

[19] V. C. Liu, P. P. Vaidyanathan, Circulant and skew circulant matrices as new normal-form realization of IIR digital filters, IEEE Trans. Circuits and Systems, 35 (1988), 625-635. 1

[20] F. L. Lu, Z. L. Jiang, The sum and product of Fibonacci numbers and Lucas numbers, Pell numbers and Pell-Lucas numbers representation by matrix method, Wseas Trans. Math., 12 (2013), 449-458. 1

[21] M. J. Narasimha, Linear convolution using skew-cyclic convolutions, IEEE Signal Process. Lett., 14 (2007), 173-176. 1

[22] S. Pelletier, J. R. Cooperstock, Preconditioning for edge-preserving image super resolution, IEEE Trans. Image Process., 21 (2012), 67-79. 1

[23] J. Shao, Z. W. Zheng, F. W. Meng, Oscillation criteria for fractional differential equations with mixed nonlinearities, Adv. Difference Equ., 2013 (2013), 9 pages. 1

[24] S.-Q. Shen, J.-M. Cen, Y. Hao, On the determinants and inverses of circulant matrices with Fibonacci and Lucas numbers, Appl. Math. Comput., 217 (2011), 9790-9797. 1 
[25] S. Solak, On the norms of circulant matrices with the Fibonacci and Lucas numbers, Appl. Math. Comput., 160 (2005), 125-132. 1

[26] Y. G. Sun, F. W. Meng, Interval criteria for oscillation of second-order differential equations with mixed nonlinearities, Appl. Math. Comput., 198 (2008), 375-381. 1

[27] J. Wang, F. W. Meng, Interval oscillation criteria for second order partial differential systems with delays, J. Comput. Appl. Math., 212 (2008), 397-405.

[28] R. Xu, F. W. Meng, Some new weakly singular integral inequalities and their applications to fractional differential equations, J. Inequal. Appl., 2016 (2016), 16 pages. 1

[29] Y. Yazlik, N. Taskara, On the norms of an r-circulant matrix with the generalized k-Horadam numbers, J. Inequal. Appl., 2013 (2013), 8 pages. 2

[30] Y. P. Zheng, S. G. Shon, Exact determinants and inverses of generalized Lucas skew circulant type matrices, Appl. Math. Comput., 270 (2015), 105-113. 1 